\title{
Incidental finding of subclavian artery occlusion and subsequent hypoplastic internal mammary artery as a candidate recipient vessel in DIEP flap breast reconstruction
}

\author{
Ik Hyun Seong, Kyong-Je Woo \\ Department of Plastic and Reconstructive Surgery, Ewha Womans University Mokdong Hospital, Ewha Womans University College of \\ Medicine, Seoul, Korea
}

We report a case of autologous breast reconstruction in which a thoracodorsal vessel was used as a recipient vessel after a hypoplastic internal mammary vessel was found on preoperative computed tomography (CT) angiography. A 46-year-old woman with no underlying disease was scheduled to undergo skin-sparing mastectomy and breast reconstruction using a deep inferior epigastric artery perforator flap. Preoperative CT angiography showed segmental occlusion of the right subclavian artery with severe atherosclerosis and calcification near the origin of the internal mammary artery, with distal flow maintained by collateral branches. The thoracodorsal artery was selected to be the recipient vessel because CT showed that it was of adequate size and was not affected by atherosclerosis. The patient experienced no postoperative complications, and the flap survived with no vascular complications. The breasts were symmetrical at a 6-month follow-up. This case highlights that preoperative vascular imaging modalities may help surgeons avoid using diseased vessels as recipient vessels in free flap breast reconstructions.

Keywords Breast reconstruction / Free flap / Subclavian artery / Internal mammary artery / Atherosclerosis
Correspondence: Kyong-Je Woo Department of Plastic and

Reconstructive Surgery, Ewha Womans University Mokdong Hospital, Ewha Womans University College of Medicine, 1071

Anyangcheon-ro, Yangcheon-gu,

Seoul 07985, Korea

Tel: +82-2-2650-5149

Fax: +82-2-2650-5278

E-mail: economywoo@gmail.com

Received: March 29, $2019 \bullet$ Revised: May 23, $2019 \bullet$ Accepted: June 12, 2019

pISSN: 2234-6163 • elSSN: 2234-6171 • https://doi.org/10.5999/aps.2019.00430 • Arch Plast Surg 2019;46:599-602

\section{INTRODUCTION}

In autologous tissue-based breast reconstruction, the deep inferior epigastric artery perforator (DIEP) flap has become the gold standard technique. According to Lie et al. [1], the failure rate of DIEP flaps is about $0 \%-3 \%$. A reliable recipient vessel is critical for avoiding flap failure. The internal mammary vessels that originate from the subclavian artery are the most common and reliable recipient vessels in breast reconstruction using a DIEP flap [2]. The antegrade internal mammary artery (IMA) and retrograde IMA are commonly used as recipient vessels $[3,4]$. In this report, we present a case of incidentally found subclavian artery occlusion and subsequent hypoplastic IMA discovered during a preoperative evaluation for autologous breast reconstruction. 


\section{CASE}

A 46-year-old woman with a body mass index of $24.34 \mathrm{~kg} / \mathrm{m}^{2}$ was scheduled to undergo skin-sparing mastectomy on her right breast and immediate breast reconstruction using a DIEP flap. She was a non-smoker and had no underlying disease such as hypertension, diabetes mellitus, or peripheral arterial occlusive disease (PAD).

On preoperative computed tomography (CT) angiography, segmental occlusion and severe atherosclerosis were identified in the right subclavian artery, with calcification near the origin of the right IMA. The distal flow of the IMA was maintained by collateral branches (Fig. 1). Although the flow was preserved by collateral branches, the right IMA was smaller than the left IMA, suggesting decreased blood flow (Fig. 2). The thoracodorsal vessels were adequate in size and were without atherosclerosis (Fig. 3); therefore, the thoracodorsal artery was chosen to be the recipient vessel.

A right skin-sparing mastectomy (360 g) was performed by the oncologic surgery team. A DIEP flap, including three ipsilateral perforators $(872 \mathrm{~g})$, was harvested, and the inset rate of the flap was $64 \%$ (562/872 g). The pedicle vessels of the flap were anastomosed to the thoracodorsal artery and vein by end-toend anastomoses. The patient experienced no immediate postoperative complications, and the flap survived without vascular complications. The breasts were symmetrical at a 6-month follow-up and showed no long-term complications. Postoperative three-dimensional photographs at 6 months confirmed symmetrical breast size. The breast volumes were 438 and $421 \mathrm{~mL}$ on the right and left, respectively (Fig. 4).

\section{Fig. 1. Segmental occlusion of the right subclavian artery}

Preoperative computed tomography angiography showed segmental occlusion of the right subclavian artery (arrows) with severe atherosclerosis near the origin of the internal mammary artery.

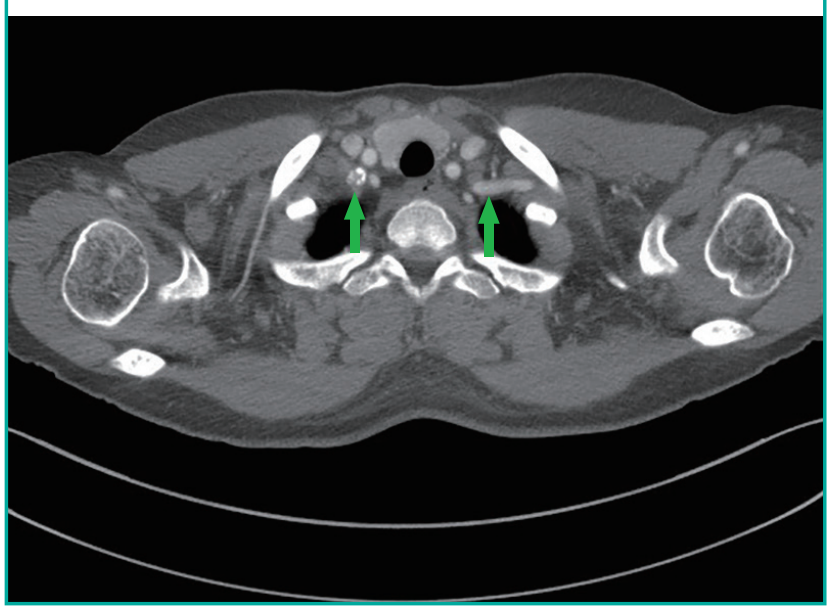

\section{DISCUSSION}

The vascular anatomy of the inferior abdominal wall can be evaluated preoperatively with CT angiography [5]. Preoperative $\mathrm{CT}$ angiography has been shown to reduce the operative time by minimizing the time spent finding perforators, selecting surgical sites, and identifying the courses of vessels [6]. Three-dimensional CT angiography can be used to estimate abdominal

\section{Fig. 2. Hypoplastic right internal mammary artery \\ On preoperative computed tomography angiography, the hypoplas- tic right internal mammary artery (arrow) was observed at the third intercostal space.}

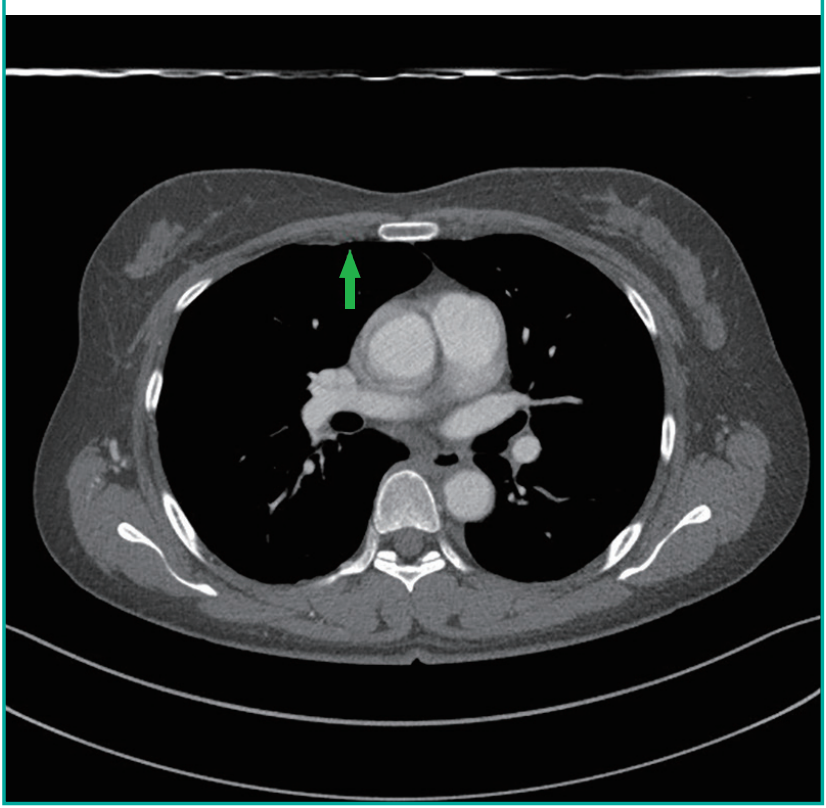

Fig. 3. Thoracodorsal artery

The thoracodorsal artery (arrows) showed adequate size and flow on preoperative computed tomography angiography, and was used as the recipient artery for deep inferior epigastric artery perforator flap breast reconstruction.

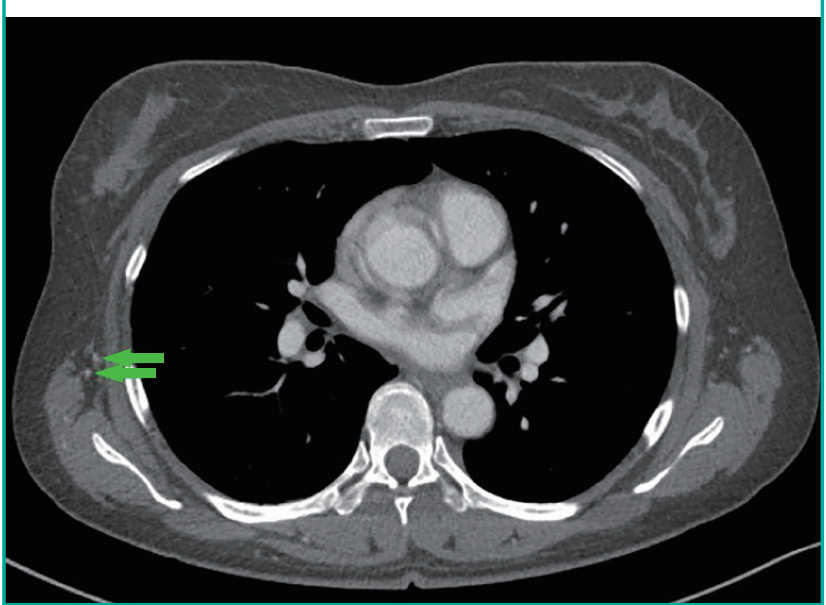




\section{Fig. 4. Preoperative and postoperative photos}

(A) Preoperative photograph. (B) Six months postoperatively. (C, D) Three-dimensional photographs at 6 months postoperatively, showing symmetrical breasts.
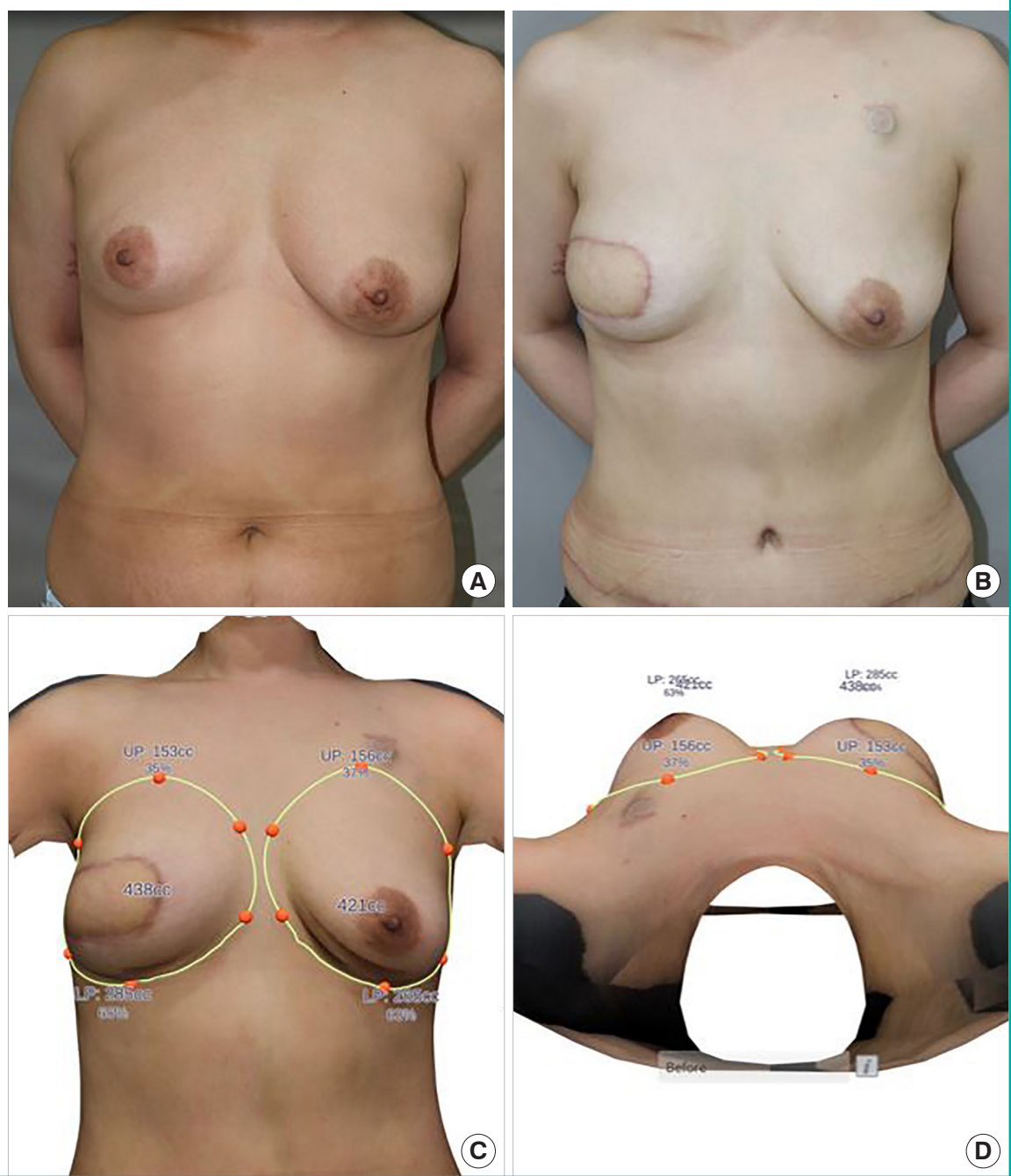

flap volume before surgery, which guides the amount of tissue to harvest $[7,8]$. Similarly, CT angiography is useful for evaluating recipient vessels [9]. In a study by Sisto and Isola [10], only $3.1 \%$ of patients were found to have notable stenosis in the IMA [10]. In addition, Shadman et al. [11] reported that approximately $2 \%$ of the independently-living population showed significant subclavian artery stenosis, which was attributed to a previous or current history of smoking, elevated systolic blood pressure, high-density lipoprotein levels (inversely), and the presence of PAD.

In previous studies using the IMA as the recipient vessel for DIEP flaps, IMA sclerosis has not been reported. In a previous study of DIEP flap cases, the IMA was found not to be suitable for vascular anastomosis in three separate cases, and the thoracodorsal artery was instead used. In two cases (one left- and one right-sided reconstruction), the conversion was performed due to friability of the vessel, and in the third case (left-sided recon- struction), the reason for the conversion was absence of the IMA [12].

The current case highlights the fact that the IMA may not always be an appropriate recipient artery because of atherosclerosis and the occlusion of proximal major arteries. Since flow is dependent on a pressure differential, stenosis of the IMA can lead to decreased blood flow and vascular complications of the flap when it is used as a recipient vessel [13]. Of note, Kropf et al. [14] reported a higher rate of fat necrosis after muscle-sparing transverse rectus abdominis myocutaneous flap reconstruction using the thoracodorsal vessels than with the internal mammary vessels. Although the exact mechanism is unknown, lower blood flow of the thoracodorsal artery compared to the IMA may be a factor [15].

The current case report demonstrated that in rare cases, the IMA might be hypoplastic because of proximal atherosclerosis, and that a preoperative CT angiographic evaluation of the re- 
cipient vessels in free flap breast reconstruction may be helpful for preventing the use of inappropriate recipient vessels.

\section{NOTES}

\section{Conflict of interest}

No potential conflict of interest relevant to this article was reported.

\section{Ethical approval}

The study was approved by the Institutional Review Board of Ewha Womans University Mokdong Hospital (IRB No. 201905-024) and performed in accordance with the principles of the Declaration of Helsinki. Written informed consent was obtained.

\section{Patient consent}

The patient provided written informed consent for the publication and the use of her images.

\section{Author contribution}

Conceptualization: Woo KJ. Methodology: Woo KJ. Data curation: Seong IH. Writing-original draft: Seong IH. Writing-review \& editing: Woo KJ.

\section{ORCID}

Ik Hyun Seong https://orcid.org/0000-0003-0289-0222

Kyong-Je Woo https://orcid.org/0000-0002-7349-6743

\section{REFERENCES}

1. Lie KH, Barker AS, Ashton MW. A classification system for partial and complete DIEP flap necrosis based on a review of 17,096 DIEP flaps in 693 articles including analysis of 152 total flap failures. Plast Reconstr Surg 2013;132:1401-8.

2. Hefel L, Schwabegger A, Ninkovic M, et al. Internal mammary vessels: anatomical and clinical considerations. $\mathrm{Br} \mathrm{J}$ Plast Surg 1995;48:527-32.

3. Li S, Mu L, Li Y, et al. Breast reconstruction with the free bipedicled inferior TRAM flap by anastomosis to the proximal and distal ends of the internal mammary vessels. J Reconstr Microsurg 2002;18:161-8.

4. Mackey SP, Ramsey KW. Exploring the myth of the valve- less internal mammary vein: a cadaveric study. J Plast Reconstr Aesthet Surg 2011;64:1174-9.

5. Clavero JA, Masia J, Larranaga J, et al. MDCT in the preoperative planning of abdominal perforator surgery for postmastectomy breast reconstruction. AJR Am J Roentgenol 2008;191:670-6.

6. Tong WM, Dixon R, Ekis H, et al. The impact of preoperative CT angiography on breast reconstruction with abdominal perforator flaps. Ann Plast Surg 2012;68:525-30.

7. Rosson GD, Shridharani SM, Magarakis M, et al. Three-dimensional computed tomographic angiography to predict weight and volume of deep inferior epigastric artery perforator flap for breast reconstruction. Microsurgery 2011;31: 510-6.

8. Woo KJ, Kim EJ, Lee KT, et al. A novel method to estimate the weight of the DIEP flap in breast reconstruction: DIEPW, a simple calculation formula using paraumbilical flap thickness. J Reconstr Microsurg 2016;32:520-7.

9. Kim H, Lim SY, Pyon JK, et al. Preoperative computed tomographic angiography of both donor and recipient sites for microsurgical breast reconstruction. Plast Reconstr Surg 2012;130:11e-20e.

10. Sisto T, Isola J. Incidence of atherosclerosis in the internal mammary artery. Ann Thorac Surg 1989;47:884-6.

11. Shadman R, Criqui MH, Bundens WP, et al. Subclavian artery stenosis: prevalence, risk factors, and association with cardiovascular diseases. J Am Coll Cardiol 2004;44:618-23.

12. O’Neill AC, Hayward V, Zhong T, et al. Usability of the internal mammary recipient vessels in microvascular breast reconstruction. J Plast Reconstr Aesthet Surg 2016;69:90711.

13. Tomioka YK, Uda H, Yoshimura K, et al. Studying the blood pressures of antegrade and retrograde internal mammary vessels: Do they really work as recipient vessels? J Plast Reconstr Aesthet Surg 2017;70:1391-6.

14. Kropf N, Macadam SA, McCarthy C, et al. Influence of the recipient vessel on fat necrosis after breast reconstruction with a free transverse rectus abdominis myocutaneous flap. Scand J Plast Reconstr Surg Hand Surg 2010;44:96-101.

15. Lorenzetti F, Kuokkanen H, von Smitten K, et al. Intraoperative evaluation of blood flow in the internal mammary or thoracodorsal artery as a recipient vessel for a free TRAM flap. Ann Plast Surg 2001;46:590-3. 